\title{
A Comparison of Second-Order and High-Order of Finite Difference Staggered-Grid Method in 2D P-SV Wave Propagation Modelling using Graphics Processing Unit
}

\author{
Irchan Bani Hasym ${ }^{1}$, Sudarmaji ${ }^{2}$, Sismanto $^{3}$, and Waluyo ${ }^{4}$ \\ Geophysics Sub Department, Department of Physics, Faculty of Mathematics and Natural Sciences \\ Gadjah Mada University \\ Yogyakarta, Indonesia \\ ${ }^{1}$ irchanbani@gmail.com, ${ }^{2}$ ajisaroji@ugm.ac.id, ${ }^{3}$ sismanto@ugm.ac.id , ${ }^{4}$ walgeof@yahoo.com
}

\begin{abstract}
A comparison of second order and high order $\left(4^{\text {th }}, 8^{\text {th }}\right.$ and $12^{\text {th }}$ order $)$ of finite difference staggered-grid method on 2D P-SV elastic wave propagation has been conducted. The comparison plot of numerical dispersion and accuracy among each order has been generated to show the stability condition. CUDA $C$ programming language is used to apply the finite difference staggeredgrid schema on GPU NVIDIA GeForce GTX-590 and to measure the duration of process time quantitatively. The code has been verified by comparing travel time of direct wave, reflection, refraction and conversion from the synthetic seismogram with analytical calculation from two layer medium using Snell's law. The program is applied to one-layer medium and complex medium (Marmousi-2 model). The time process of one shot simulation on Marmousi-2 model with $4800 \times 2400$ grid size are $1168.72 \mathrm{~s}\left(2^{\text {nd }}\right.$ order $), 1736.40 \mathrm{~s}\left(4^{\text {th }}\right.$ order $)$, $2816.80 \mathrm{~s}\left(8^{\text {th }}\right.$ order $)$ and $3778.67 \mathrm{~s}\left(12^{\text {th }}\right.$ order $)$. The difference of seismogram showed that the second order and high order of the finite difference on two layer model indicate that the numerical dispersion has occurred on the waveform. The result shows that the higher order of the finite difference will have less numerical dispersion compared to the low order but need longer process time.
\end{abstract}

Keywords: elastic wave, finite difference, high order, GPU, CUDA, numerical dispersion, accuracy

\section{INTRODUCTION}

Finite-difference methods (FDM) are widely used in seismic wave modelling [1-3]. The FDM have a straightforward implementation to code, and capable to handle very complex seismic model. The weakness of FDM is that the accuracy of this method depends on the order of accuracy of the FDM [4]. FDM are often used in conjunction with perfectly matched layer (PML) to absorb waves on the artificial edges of the numerical grid to mimic an infinite or semi-infinite medium [5] or convolutional PML [6].

In recent years, the development of wave modelling is significantly increased along with the usage of Graphics Processing Unit (GPU) to accelerate computation time. GPU programming has become easier with the introduction of CUDA $\mathrm{C}$ because its syntax is similar to $\mathrm{C}$ languange program.

In this study, we use GPU to accelerate computation time in the simulation of wave propagation. In addition to that we compare the degree of accuracy between secondorder from [7] and high-order $\left(4^{\text {th }}, 8^{\text {th }}\right.$ and $12^{\text {th }}$ order) based on synthetic seismogram result, seismic data record, and snapshot of wave propagation through the model.

\section{FORMULATION}

We use a 2D medium with $x$ and $z$ axis. The medium is assumed linearly elastic and isotropic.

\section{A. Equations}

Basis of the P-SV seismic wave modelling is velocitystress equation [2] which are :

$$
\begin{aligned}
\rho \frac{\partial v_{x}}{\partial t} & =\frac{\partial \tau_{x x}}{\partial x}+\frac{\partial \tau_{x z}}{\partial z} \\
\rho \frac{\partial v_{z}}{\partial t} & =\frac{\partial \tau_{x z}}{\partial x}+\frac{\partial \tau_{z z}}{\partial z} \\
\frac{\partial \tau_{x x}}{\partial t} & =(\lambda+2 \mu) \frac{\partial v_{x}}{\partial x}+\lambda \frac{\partial v_{z}}{\partial z} \\
\frac{\partial \tau_{z z}}{\partial t} & =(\lambda+2 \mu) \frac{\partial v_{z}}{\partial z}+\lambda \frac{\partial v_{x}}{\partial x} \\
\frac{\partial \tau_{x z}}{\partial t} & =\mu\left(\frac{\partial v_{x}}{\partial z}+\frac{\partial v_{z}}{\partial x}\right)
\end{aligned}
$$

Where $\left(v_{x}, v_{z}\right)$ is the velocity vector, and $\left(\tau_{x x}, \tau_{z z}, \tau_{x z}\right)$ is the stress tensor. $\rho(x, z)$ is density and $\lambda(x, z)$ and $\mu(x, z)$ are Lamé coefficient.

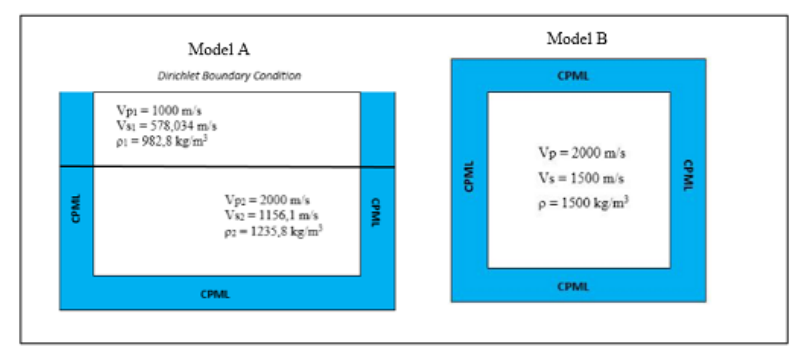

Figure 1. Elastic Parameter of Model A and Model B 


\section{B. Model}

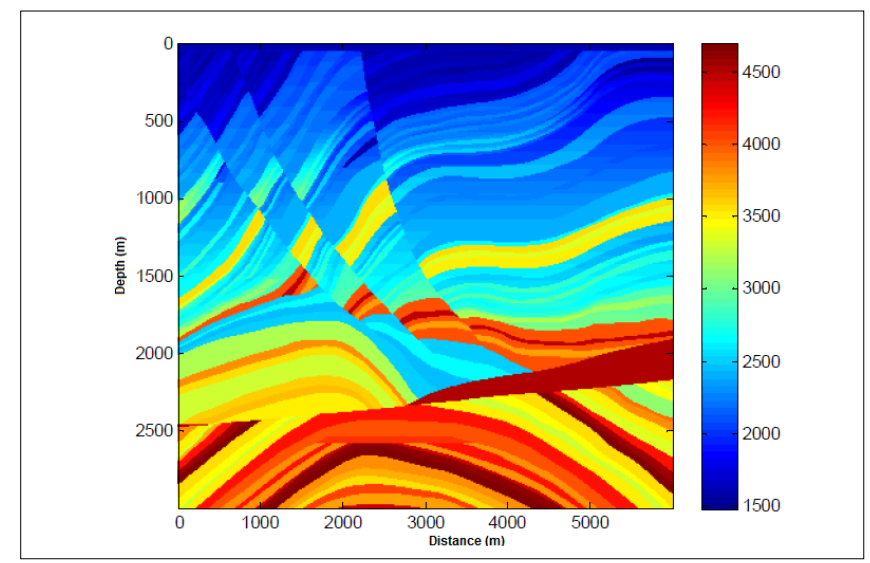

Figure 2. P-wave velocity of Model C.

In this study, we use three models to compare secondorder with high order. First, model A is a semi-infinite two layer elastic model that will be used to verify the logic of the code. Second, model B is an infinite elastic model (Fig. 1). Lastly, model $\mathrm{C}$ is a slice from distance 8000 to $14000 \mathrm{~m}$ of Marmousi2 model (Fig. 2). The Marmousi2 model which is an elastic upgrade from Marmousi model was created by [8].

\section{Boundary Conditions}

Dirichlet condition or rigid-surface condition is applied in surface boundary (top) of the models to generate surfacewave. Meanwhile, absorbing boundary using Convolutional Perfectly Matched Layer (CPML) is applied in left, right, and bottom of the models. Yet absorbing boundary is used in all side of model B for generating a reflection less snapshot. The thickness of CPML that applied to each model is 10 grid points.

\section{Source Excitation}

Ricker wavelet is used as a source of the models. This source is applied to vertical stress component. Mathematical formula for a Ricker Wavelet [9] is given by :

$$
\text { Ricker }(t)=\left(1-2 \pi^{2} f^{2} t^{2}\right) e^{\pi^{2} f^{2} t^{2}}
$$

Each of models has a different dominant frequency applied to them that is $7 \mathrm{~Hz}, 45 \mathrm{~Hz}$, and $20 \mathrm{~Hz}$ for Model A, B, and $\mathrm{C}$, respectively. This difference affects the number of point per wavelength and the numerical dispersion.

\section{NUMERICAL ANALYSIS}

\section{A. Finite-Difference Equation}

Finite-difference staggered-grid method is a numerical solution using derivative equations within a grid that separate the velocity and the stress node position. In this study, we use a constant $2^{\text {nd }}$-order in time and a different order in space. In other words we use Explicit Finite Difference Time
Domain (EFDTD) staggered-grid. Referring to [10] the following expression is used to calculate different-order in

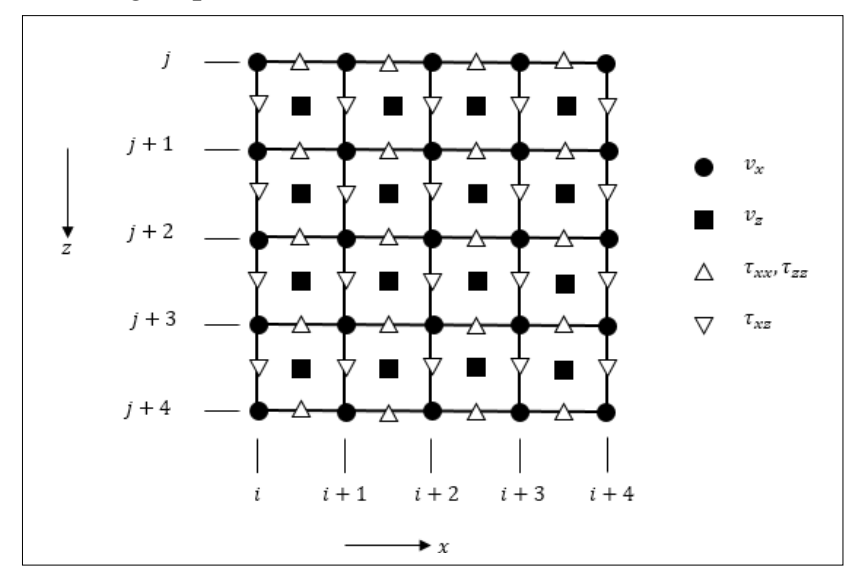

Figure 3. Discretization of the medium on a staggered-grid (modified from [2]).

the first order spatial derivative:

$\frac{\partial f}{\partial x}=\frac{1}{\Delta x} \sum_{n=1}^{N} C_{n}^{(N)}\left\{\begin{array}{c}f\left[x+\frac{\Delta x}{2}(2 n-1)\right]- \\ f\left[x-\frac{\Delta x}{2}(2 n-1)\right]\end{array}\right\}+O\left(\Delta x^{2 N}\right)$

Where $N$ is a value from $2 \mathrm{~N}$-order approximation and $\Delta x$ is the grid steps for the $x$-axis. The key point in (3) is to compute the difference coefficient $C_{n}^{(N)}$. This coefficient is obtained using algorithm from [11][12].

TABLE 1. COEFFICIENT OF EFDTD METHOD

\begin{tabular}{|c|c|c|c|c|c|c|}
\hline Orde & C1 & C2 & C3 & C4 & C5 & C6 \\
\hline 2 & 1 & & & & & \\
\hline 4 & $\frac{9}{8}$ & $-\frac{1}{24}$ & & & & \\
\hline 8 & $\frac{1715}{1434}$ & $-\frac{114}{1434}$ & $\frac{14}{1434}$ & $-\frac{1}{1434}$ & & \\
\hline 12 & $\frac{55902}{45771}$ & $-\frac{4437}{45771}$ & $\frac{799}{45771}$ & $-\frac{136}{45771}$ & $\frac{16}{45771}$ & $-\frac{1}{45771}$ \\
\hline
\end{tabular}

\section{B. Stability}

Stability equation for elastic medium can be obtained by changing the parameter of anisotropic stability equation[10]. So the following expression is used for $\operatorname{EFDTD}\left(\Delta \mathrm{t}^{2}, \Delta \mathrm{x}^{2 \mathrm{~N}}\right)$ :

$$
\Delta t \sqrt{\frac{V_{p}^{2}}{\Delta x^{2}}+\frac{V_{s}^{2}}{\Delta z^{2}}} \leq \frac{1}{d}
$$

Where, $d=\sum_{n=1}^{N} C_{n}^{(N)}(-1)^{n-1}$. The stability value $(1 / d)$ of each model is same, but the time step is different. This is occurred because the difference of $\mathrm{P}$ and $\mathrm{S}$ wave velocity, and also grid step $(\Delta x)$. The choice of high-order time step depends on the choice of second-order time step by [7]. The difference between time step maximum and time step on second-order is used to calculate time step in high-order. Detailed information about stability value in each model and order accuracy is described in Ref [13]. 


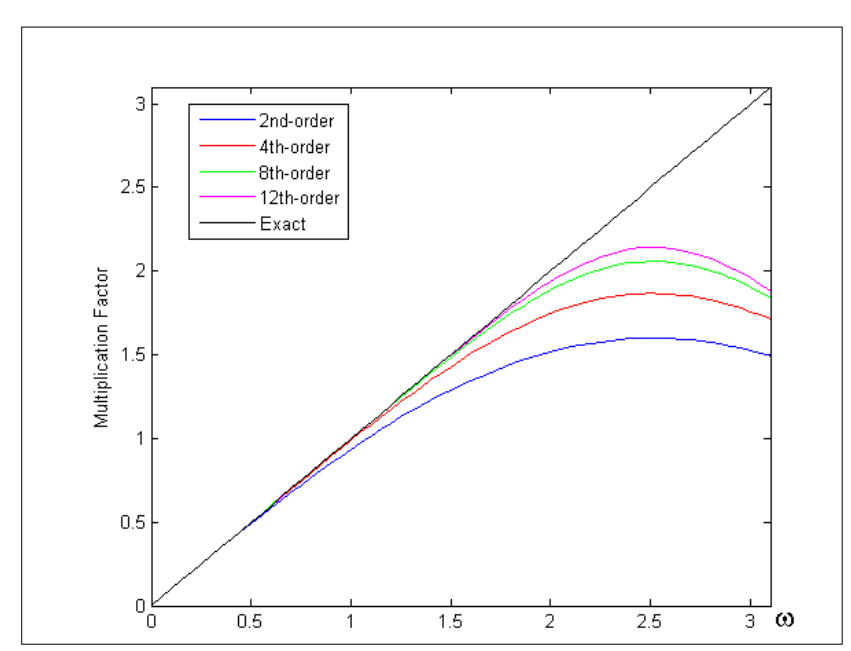

Figure 4. Plots of exact $\omega$ and EFDTD method in different orders number.

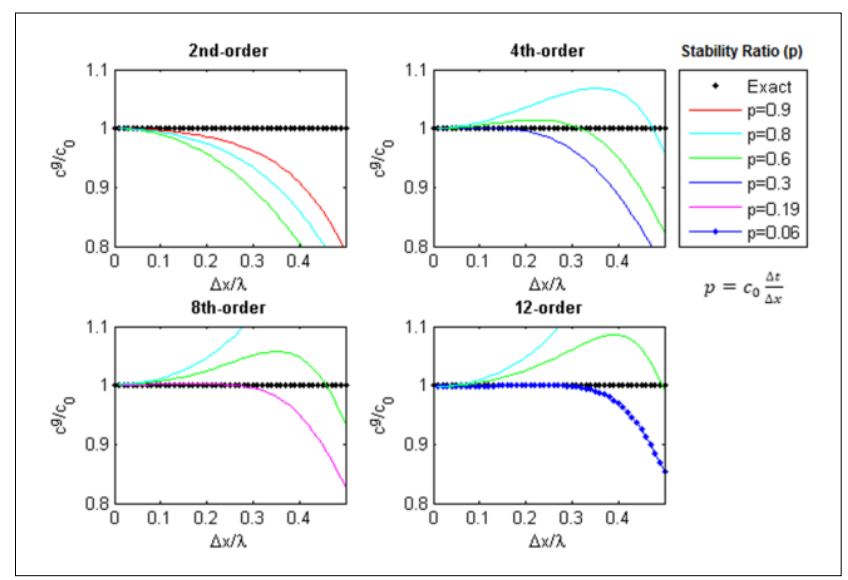

Figure 5. Plot of EFDTD method dispersion curves of 1D elastic wave equation modelling.

\section{Accuracy}

Comparison of the EFDTD accuracy with the exact value can be obtained by knowing the exact derivative of $e^{i \omega x}[14]$. It is shown by the following expression:

$$
\frac{d}{d x}\left(e^{i \omega x}\right)=i \omega e^{i \omega x}=\omega\left(i e^{i \omega x}\right)
$$

Yet the numerical derivative of $e^{i \omega x}$ for several order numbers of EFDTD method [12] :

$$
\frac{\partial f}{\partial x}=\frac{2 \sum_{n=1}^{N} C_{\mathrm{n}}^{(\mathrm{N})} \sin \left(\frac{\omega(2 n-1) \Delta x}{2}\right)}{\Delta x}\left(i e^{i \omega x}\right)
$$

Now, we can obtain the EFDTD accuracy by comparing factor of $\omega$ and $\left[2 \sum_{n=1}^{N} C_{\mathrm{n}}^{(\mathrm{N})} \sin (\omega(2 n-1) \Delta x / 2)\right] / \Delta x$. It is shown in Fig. 4 as exact line and several order lines. Fig. 4 indicated that the accuracy of EFDTD increases with increasing order numbers.

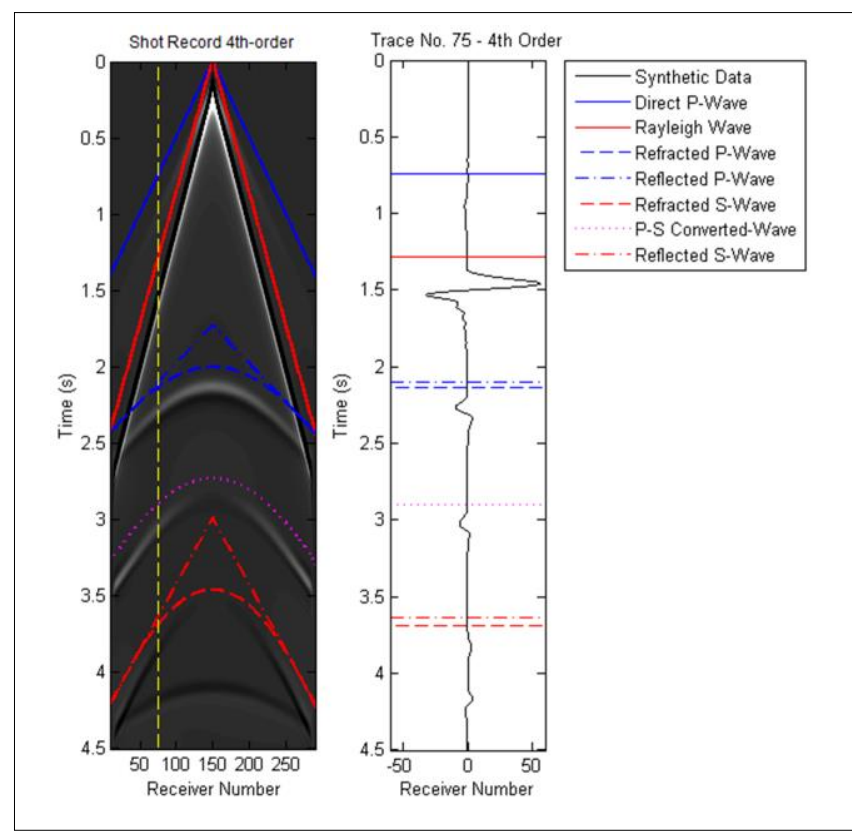

Figure 6. Verification of synthetic seismogram using Snell's law calculation.

\section{Dispersion}

Description about the second-order of EFDTD dispersion can be shown by using 1D elastic EFDTD equation [15]. So the following expression is the modified equation to handle the several orders of EFDTD dispersion:

$c^{\text {grid }}=\frac{\omega}{k}=\frac{\Delta x}{\pi \Delta t} \frac{\lambda}{\Delta x} \arcsin \left(p \sum_{n=1}^{N} C_{\mathrm{n}}^{(\mathrm{N})} \sin \left(\frac{\pi(2 n-1) \Delta x}{\lambda}\right)\right)(7)$

with $p=c_{0} \Delta t / \Delta x$ and $c_{0}$ is a phase velocity of homogenous medium. The dispersion relation that shown in Fig.5 is a relation between $c^{\text {grid }} / c_{0}$ and $\Delta x / \lambda$. From Fig. 5, we obtain the minimum spatial sampling to minimize the numerical dispersion. The spatial sampling required is at least $10,5,3.5$, and 3 for $2^{\text {nd }}, 4^{\text {th }}, 8^{\text {th }}$ and $12^{\text {th }}$ order, respectively.

\section{PARALLELIZATION}

\section{A. Graphics Processing Unit}

Graphics Processing Unit is computer hardware to calculate a complex graphics data. GPUs are optimized for 2D graphics image processing. Programming on GPU that called GPGPU (General-purpose Programming on Graphics Processing Unit) is a method to accelerate non-graphics applications using GPU. GPU consists of hundreds of streaming processor cores [16]. GPU NVIDIA GeForce GTX 590 is used in this study offer computing solutions in the form of GPGPUs. It is consists of 2 GPU. One GPU has specifications: 1535.69 Megabyte Global Memory, 16 multiprocessor and 512 cores. One parallel calculation along $32 \times 16$ or 512 thread needs 10 time calculation to cover the entire matrix [7]. 


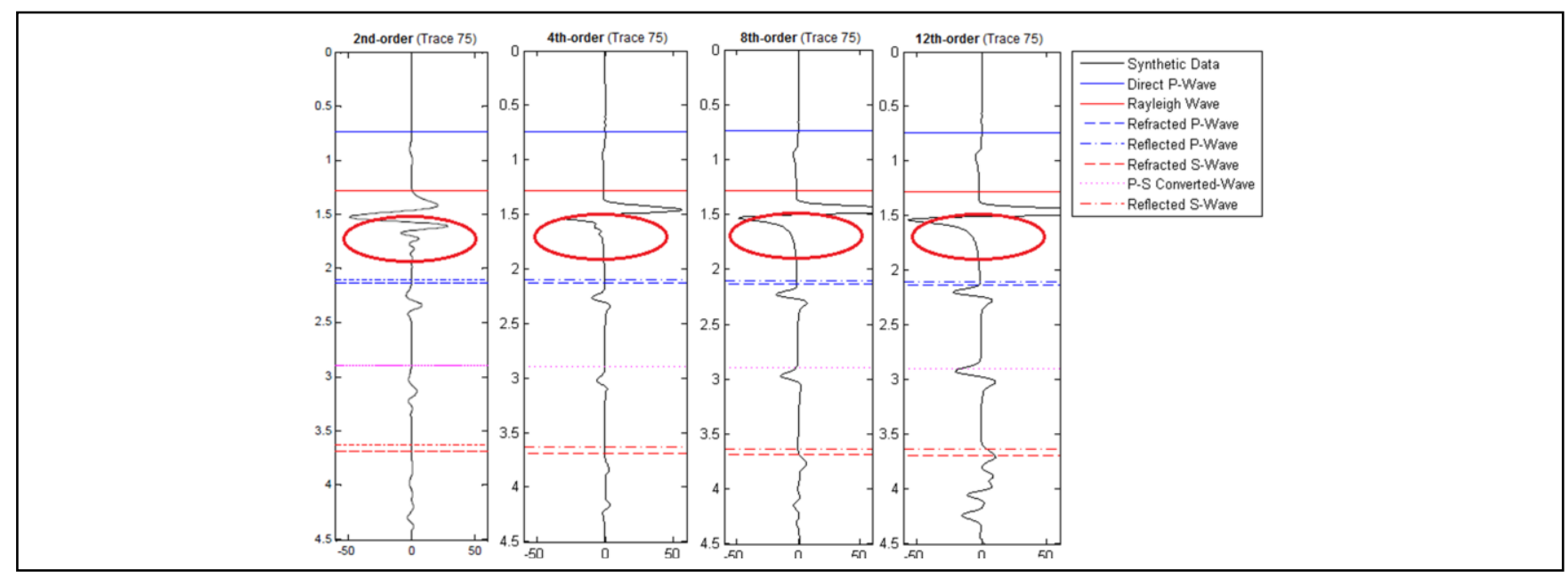

Figure 7. Comparison of synthetic seismogram of Model A with different orders.

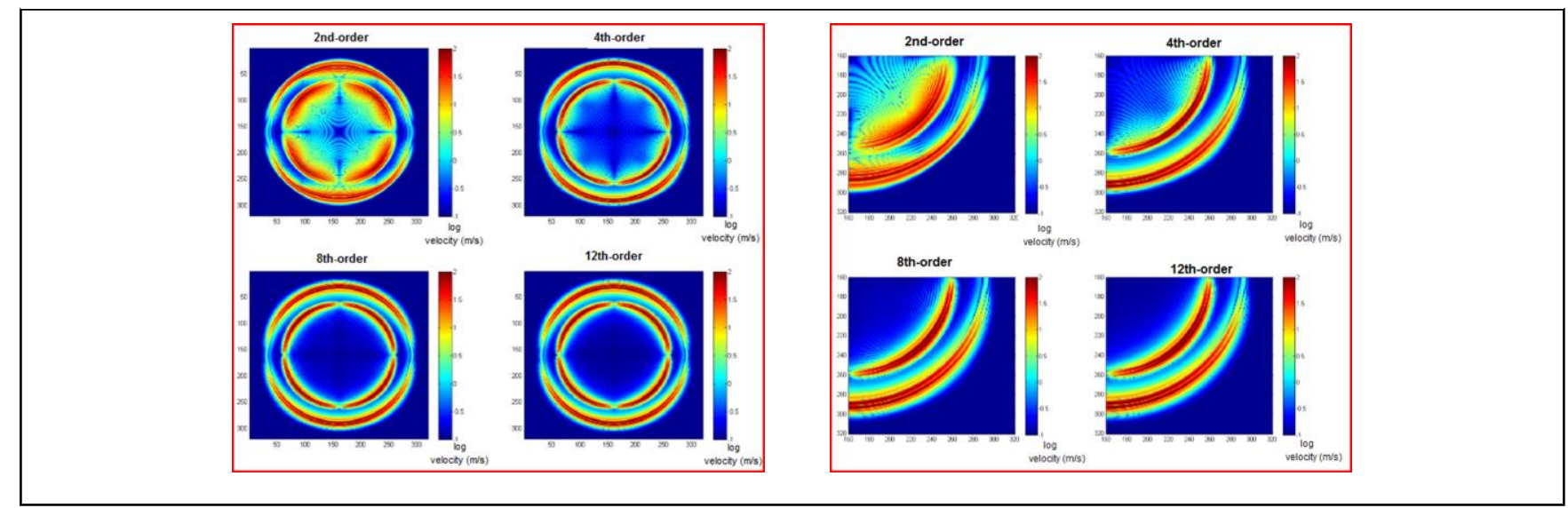

Figure 8. Comparison of wave propagation snapshot of Model B; (a) normal scale (b) zooming scale
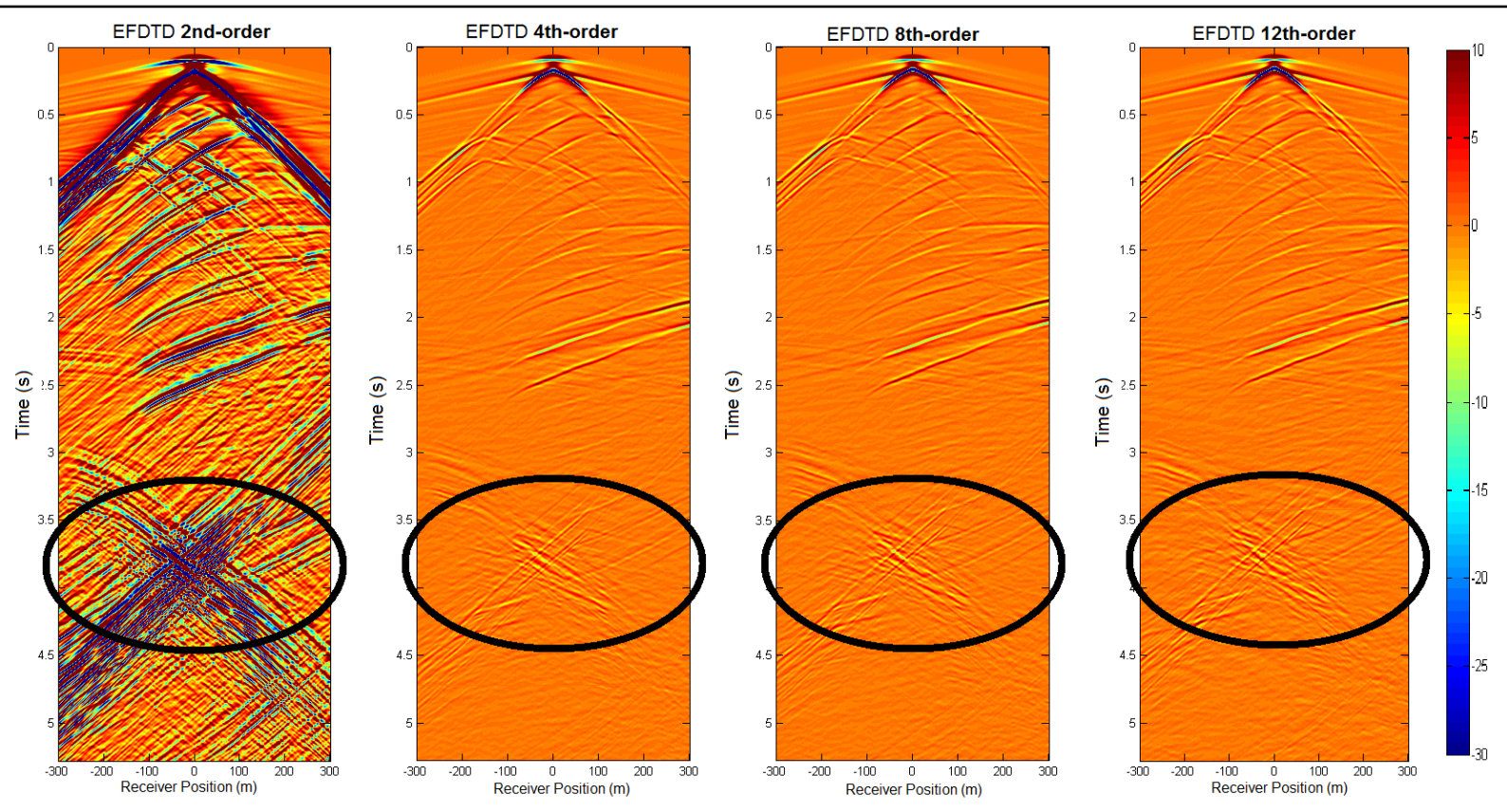

Figure 9. Comparison of seimic data records between second-order and high-order. 


\section{B. Cuda Programming}

The CUDA (Compute Unified Device Architecture) is a programming model which can be porting to GPU. It omits hardware complexities and simplifies novice programmer work. The unit of program that is executed on the GPU is called the Kernel. This programming model completes data calculation using parallelization [16].

\section{RESULTS AND DISCUSSION}

\section{A. Code Verification}

The code of high-order program is verified by travel time of direct wave, reflection, refraction and conversion from the synthetic seismogram with analytical calculation from two layer medium using Snell's law as in [7]. The synthetic seismogram which is one of the simulation result has a good agreement with the analytical line [6]. So the code can be applied to handle Model B and very complex model like Model C.

\section{B. Comparison of Time Process}

Time process data between second-order and high-order is obtained from the same condition. These conditions include the grid size of model, the model, and the time step. The result of this comparison shows a description of the different time length that needed to finish the simulation using GPU. Table 2 and 3 show that longer time is needed to finish the wave propagation simulation with increasing order number.

TABLE 2. COMPARISON OF TIME PROCESS ON MODEL B

\begin{tabular}{|c|c|c|c|c|c|}
\hline \multirow{2}{*}{ Grid Size } & \multirow{2}{*}{$\begin{array}{c}\text { The Amount of } \\
\text { Element }\end{array}$} & \multicolumn{4}{|c|}{ Time Process (s) } \\
\cline { 3 - 6 } & $\begin{array}{c}\mathbf{2}^{\text {nd }} \\
\text { order }\end{array}$ & $\begin{array}{c}\mathbf{4}^{\text {th }} \\
\text { order }\end{array}$ & $\begin{array}{c}\mathbf{8}^{\text {th }} \\
\text { order }\end{array}$ & $\begin{array}{c}\mathbf{1 2}^{\text {th }} \\
\text { order }\end{array}$ \\
\hline $320 \times 320$ & 102400 & 0.36 & 0.43 & 0.56 & 0.74 \\
\hline $640 \times 640$ & 409600 & 1.33 & 1.61 & 2.23 & 2.85 \\
\hline $1280 \times 1280$ & 1638400 & 5.26 & 6.46 & 8.9 & 11.22 \\
\hline $2560 \times 2560$ & 6553600 & 21.48 & 25.99 & 36.24 & 44.97 \\
\hline $4000 \times 4000$ & 16000000 & 54.75 & 63.7 & 87.72 & 109.85 \\
\hline
\end{tabular}

TABLE 3. COMPARISON OF TIME PROCESS ON MODEL C

\begin{tabular}{|c|c|c|c|c|c|}
\hline $\begin{array}{c}\text { Order of } \\
\text { Accuracy }\end{array}$ & $\begin{array}{c}\text { Time } \\
\text { Step }(\Delta \boldsymbol{t})\end{array}$ & Step & Duration & $\begin{array}{c}\text { Time } \\
\text { Process } \\
\text { in 1 } \\
\text { shot }\end{array}$ & $\begin{array}{c}\text { Duration } \\
\mathbf{( 5 . 2 8 ~ s )}\end{array}$ \\
\hline $2^{\text {nd }}$-order & 0.000176 & 30000 & 5.28 & 1168.72 & 1168.72 \\
\hline $4^{\text {th }}$-order & 0.000143 & 37000 & 5.29 & 1740.02 & 1736.40 \\
\hline $8^{\text {th }}$-order & 0.000125 & 42500 & 5.31 & 2834.14 & 2816.80 \\
\hline $12^{\text {th }}$-order & 0.000118 & 45000 & 5.31 & 3800.14 & 3778.67 \\
\hline
\end{tabular}

Furthermore, $12^{\text {th }}$-order needs four time process than second-order to finish the wave propagation simulation on a same duration. This difference is caused by the different node number calculated from the iteration. The amount of node accords with order value that is used. So the node number calculated is $2,4,8,12$ for each order that is used in this study.

\section{Comparison of EFDTD Accuracy}

The amount of trailing wave that appears on the model is used to find the level difference of accuracy between secondorder and high-order. Trailing wave is an expression of the numerical dispersion. Parameter value of the simulation is made on a same condition to give a valid result for each model. Those parameters are frequency, grid step, grid size, and time duration. Figure 7 shows that the trailing wave decreases with increasing order number. In particular marked that marked by red ellipse, it shows the smoothness of Rayleigh wave tail in high-order EFDTD method. On the contrary, the accuracy of EFDTD method increases with increasing order number. Unfortunately, the bottom of the synthetic seismogram shows a strong secondary reflection from absorbing layer (blue circle). It indicates that the thickness of absorbing layer needs to be increased with increasing order-number. This problem occurs because the amount of the calculated node number is increase. Fig. 8 shows that the wave propagation snapshot of high-order much clearer than second-order. Seismic data records from Model $C$ that use $4800 \times 2400$ grid size and 1.25 grid step show a great accuracy level for high-order EFDTD method. It also indicates that the accuracy between fourth-order and another high-order has not a contrast different in big scale as shown in Figure 9.

\section{CONCLUSION}

We have made codes and programs of EFDTD method to simulate P-SV wave propagation using GPU. Model C programs of $12^{\text {th }}$-order need four time process than secondorder to finish the wave propagation simulation on a same duration. The wave propagation snapshots of high-order are much clearer than second-order. Each of the comparison results shows that the higher order of the finite difference have less numerical dispersion compared to the low order but need longer process time.

\section{REFERENCES}

[1] Alterman, Z.S., and Karal Jr., F.C., Propagation of Elastic Waves in Layered Media by Finite Difference Methods, Bulletin of the Seismological Society of America, Vol. 58, No. 1, Feb. 1968, pp. 367-398.

[2] Virieux, J., P-SV Wave Propagation in Heteregenous Media : Velocity-Stress Finite-Difference Method, Geophysics, Vol. 51, No.4, April 1986, pp. 889-899.

[3] Levander, A.R., Fourth-Order Finite-Difference P-SV Seismogram, Geophysics, Vol. 53, No. 1, Nov. 1988, pp. 1425-1436.

[4] Zakaria, A., Numerical Modelling of Wave Propagation Using Higher Order Finite-Difference Formulas, Thesis, Curtin University of Technology, Bentley, May 2003.

[5] Berenger, J.P., A Perfecftly Matched Layer for The Absorption of Electromagnetic Waves. Journal of Computational Physics, Vol. 114, 1994, pp. 185-200.

[6] Komatitsch, D., and Martin, R., An Unsplit Convolutional Perfectly Matched Layer Technique 
Improved at Grazing Incidence for the Seismic Wave Equation, Geophysics, Vol. 72, No. 5, 2007, pp. SM155-SM167.

[7] Dinendra, L.C., Akselerasi Perhitungan Numerik Penjalaran Gelombang P-SV Metode Finite difference Menggunakan Graphics Processing Unit dengan Contoh Kasus Simulasi Survei Seismik 2D, Undergraduate Thesis, Faculty of Mathemarics and Natural Sciences, Gadjah Mada University, 2013.

[8] Martin, G.S., The Marmousi2 Model, Elastic Synthetic Data, and an Analysis of Imaging and AVO in a Structurally Complex Environment, Thesis, Faculty of the Department of Geosciences, University of Houston, Houston, May 2004.

[9] Ryan, H., Ricker, Ormsby, Klauder, Butterworth - A Choice of Wavelets, Canadian Society of Explorations Geophysicists Recorder, Sept. 1994, pp. 8-9.

[10] Dong, L.G., Ma, Z.T., Cao, J.Z., Stability of The Staggered-Grid High-Order Difference Method for First Order Elastic Wave Equation, Chinese Journalof Geophysics, Vol. 43, No. 6, 2000, pp. 904-913.

[11] Fornberg, B., Generation of Finite Difference Formulas on Arbitrarily Spaced Grids, Mathematics of Computation, Vol. 51, No. 184, Oct. 1988, pp. 699-706.

[12] Fornberg, B., Calculation of Weights in Finite Difference Formulas, Society for Industrial and Applied Mathematics, Vol. 40, No. 3, Sep. 1998, pp. 685-691.

[13] Bani Hasym, I., Perbandingan antara Orde-2 dan Ordetinggi pada Metode Finite-Difference Staggered-Grid dalam Pemodelan Penjalaran Gelombang P-SV 2D Menggunakan Graphics Processing Unit. Undergraduate Thesis, Faculty of Mathemarics and Natural Sciences, Gadjah Mada University, 2013.

[14] Ghrist, M.L., High-Order Finite Difference Methods for Wave Equations, Thesis, University of Colorado, Colorado, 2000.

[15] Mozco, P., Kristek, J., and Halada, L., The Finite Difference Method for Seismologists : An Introduction, Comenius University, Bratislava, 2004.

[16] Sivalingam, L., GPU Acceleration of a Theoretical Particle Physics Application, Thesis, University of Edinburgh, Edinburgh, Aug. 2010. 